Noname manuscript No.

(will be inserted by the editor)

\title{
Effect of a first-time interviewer on cognitive interview quality
}

\author{
Anja Mohorko • Valentina Hlebec
}

Received: date / Accepted: date

\begin{abstract}
Cognitive interviews are a useful tool for questionnaire pretesting. However, detailed information on the proper way of performing them is scarceresearchers use this method without agreement on applicable techniques or specific rules on their realization. Additionally, users of cognitive interviewing techniques only seldom explain the pretesting results in their research, leaving it to readers to trust their outcomes. Follow-up observations on the success of the interviews and the overall experience are rarely, if ever, presented. We were interested in the learnability of the techniques when performed by non-experienced, newly instructed interviewers; moreover, we wanted to understand the most common problems they had experienced. During a five-year period, 120 methodology students performed 612 cognitive interviews and analyzed 17 different survey questionnaires. The precise documentation of their assignment served as a detailed database of qualitative and quantitative information on their experiences. The results present the first-time interviewers' ability to accurately perform and analyze a cognitive interview. We show the most common mistakes and issues in all stages of the interviewing process, the influence of different interviewers' and respondents' characteristics, and the effect of the technique on the interview's success.
\end{abstract}

Keywords cognitive interview · first-time interviewers · pretesting · survey quality

\footnotetext{
A. Mohorko · V. Hlebec

Faculty of Social Sciences, University of Ljubljana,

Kardeljeva ploščad 5, 1000 Ljubljana, Slovenia

E-mail: anja.mohorko@fdv.uni-lj.si
}

This is a post-print. The final publication is available at Springer via http://dx.doi.org/10.1007/s11135-014-0081-0

Suggested citation:

Mohorko, Anja, and Valentina Hlebec. 2015. Effect of a first-time interviewer on cognitive interview quality. Quality \& Quantity 49 (5):1897-1918. doi: 10.1007/s11135-014-0081-0. 


\section{Introduction}

Pretesting is a survey design tool enabling the detection, explanation, and improvement of potential errors, and making questionnaires shorter, easier to answer, and more respondent-friendly. Consequently, measurement errors of survey data and respondent burden are reduced (Snijkers 2002).

The use of cognitive interviewing as a questionnaire pretesting method became more extensive after two seminars of Cognitive Aspects of Survey Methodology (CASM) in 1983 and 1984. Psychologists, linguists, and survey methodologists used information obtained by means of a think-aloud process to prove that mental processes influenced the survey situation to the equal amount as the actual response to the question (e.g., Jabine et al. 1984; Schwarz 2007). The idea of cognitive interviewing originated from protocol analysis, a tool used in mathematical sciences. Ericsson and Simon (1980) first suggested this type of analysis to help understand respondents' mental processes while solving mathematical problems. Results yielded in this process allow understanding of the way participants retrieve information from memory and answer questions, and the way emotions can affect responses to later questions (Jobe and Mingay 1991).

However, despite the extensive amount of research carried out on the effect of different cognitive interviewing modes (e.g., Goerman 2006; Tourangeau et al. 2000) and technique effect (e.g., Conrad and Blair 2009; DeMaio and Landreth 2004; DeMaio and Rothgeb 1996), and on quality and ways of analyzing cognitive interviewing data (e.g., Knafl et al. 2007; Rho and Sangster 2003; Snijkers 2002; Willis 2004) and context effects (e.g., Tourangeau and Rasinski 1988), there are only few studies exploring the effect of the interviewer-a vital factor in cognitive interviewing-which will be mentioned in the following paragraphs. Compared to classical interviewing, this pretesting method is still rather unknown and rarely used; therefore, specialized training is not customary and many new cognitive interviewers have to work with limited knowledge, no experience, and luck. Apart from receiving regular interviewer training, interviewers should understand the importance of pretesting; they have to comprehend and be able to correctly explain the technique to respondents, who are most probably unfamiliar with cognitive interviews; remind respondents of the technique during the interview; and record or write down respondents' answers without making them feel uncomfortable. Yet, whereas the role of the interviewer in regular surveys is often discussed, only a few studies known to the authors deal with the important topic of improving interviewer performance in cognitive interviews.

Beatty et al. (1997) performed a small-scale study ${ }^{1}$ on the effect of interviewer behavior variation on data quality. Their interviewers were experienced and had relative freedom in their behavior and in deciding which probe(s) to use with their respondents, where the probes were grouped into thematical categories after the interviews were finished. They measured the effect in behavior variation according to the type of probes used and the amount of utterances per survey question. Although their assumptions about the noticeable variation in behavior that had not been precisely specified were confirmed, they noted reasonable consistency in the patterns of behavior. However, they measured interviewer behavior exclusively

\footnotetext{
1 Three interviewers performed 17 interviews on one questionnaire.
} 
with quantitative data and technique usage (type of probes used and amount of interviewers' feedback).

In a similar research, Conrad and Blair (2009) assessed the amount of measurement error in cognitive interviewing. Highly educated interviewers with different levels of knowledge on cognitive interviewing (using two types of probes, conditional and discretionary) and four independent judges were used to analyze the agreement on problems in the questionnaire. The study discovered some discrepancies between the usages of different techniques but focused less on the effect of cognitive interviewing experience, despite having a variety of diversely experienced interviewers.

The article by Beatty and Willis (2007) is a theoretically focused research synthesis on the practice of cognitive interviewing summarizing this method's paradigms, key decisions about its study design, and possible styles of data evaluation. Unlike the above studies and many other papers employing skilled cognitive or at least regular interviewers, this and other articles by Willis $(2005,1999)$ neither assume nor require experience. He provides general suggestions on interviewer training, which-among others-consists of the following steps: familiarity with the questionnaire, knowledge of CASM, training in probing techniques, observing experienced interviewers, practice, and problem-fixing. Additionally, some of the most important skills an interviewer requires, in Willis's opinion, are "technical skills but also the ability to be flexible, spontaneous and cool under duress" (2005, italics removed), which we will also confirm in the following paragraphs.

The fact that the role and the potential (negative or positive) effects of a firsttime, inexperienced cognitive interviewer are often overlooked will be the main focus of this article. This point of view is important because it is unclear to what extent the results of a cognitive interview and, consequently, the quality of the questions are affected by the interviewer's knowledge, especially since pretesting is purposely implemented to improve a questionnaire.

Our results will be based on a vast amount of data (interviewers, respondents, and questionnaires used). We will try to answer the following research questions:

\section{How does the interviewing style of an interviewer influence the quality of a} cognitive interview?

With the help of interview transcripts and student reports, we will try to categorize and evaluate the characteristics of interviewer behavior and the interviewing process that potentially affected its final quality. In contrast to aforementioned studies, our evaluation will be based on qualitative criteria and quantitatively analyzed because of the large sample size. Our evaluation will be based on several criteria ${ }^{2}$ including the entire interviewers' work, ranging from respondent selection to the final meta-analysis and personal comments. We will also include potential demographical effects-sex and study year ${ }^{3}$ of the interviewer. The latter will serve as an objective assessment of an individual's experience level.

2. Are some techniques easier to learn/teach than others?

\footnotetext{
2 More thoroughly explained in Section 2.2 .

3 With each study year, the study curriculum includes more methodological courses and opportunities for students to expand their knowledge on surveying and improve their interviewing and analytical skills.
} 
Beginners in cognitive interviewing are often taught that the think-aloud technique requires little, if any, interviewer training, involvement, and/or knowledge about the questionnaire tested (e.g., Beatty and Willis 2007; Bolton and Bronkhorst 1996; Campanelli 2008; Willis 2005), whereas other techniques are supposedly more burdensome. Large amounts of students pretesting questionnaires with different techniques will allow us to compare the intensities of their inputted effort. To estimate the comprehensibility of the techniques, we will take into account the accuracy of their execution, the interviewers' progress in terms of quality of their work from the first interview to the last, and the frequency of swapping a technique with another one.

3. How large is the respondent effect on the quality of the results?

One of the three elements of a (survey) interview that can cause errors connected to the response effect-apart from the interviewer and the task itselfare respondents (Sudman and Bradburn 2004). In a cognitive interview, they will most probably be entirely ignorant and require a thorough explanation of what is expected of them. However, apart from their previous knowledge, some other respondent characteristics might affect the responses. In our study, we will briefly analyze the potential effects of respondents' demographical characteristics (sex, age, education, location) and the amount of their cooperation.

In this article, we therefore analyze the performance of the interviewer, the respondent effect, and their effect on the quality of cognitive interviewing techniques. Our data consist of five cognitive interviewing techniques, performed in 612 interviews by methodology students (hereinafter: interviewers) over a period of five years. This amount of material allows us to research in detail the effect of equally educated cognitive interviewers on the quality of conducted interviews, and understand the difficulties they encountered during their assignment; either is rarely, if ever, mentioned in studies where cognitive interviews were used only as a means of improving questionnaires (i.e., non-methodological studies), despite this information being crucial for understanding the actual quality of obtained data.

In our research, we will present a qualitative analysis of a wide range of collected data and compare cognitive interviewing techniques in a large amount of respondents and interviewers. Since the interviewers transcribed all the interviews and reported their thoughts on the process, we have an insight into the most detailed method study to date. By understanding the way first-time cognitive interviewers perceive these qualitative pretesting methods on a quantitative level and how successfully they grasp them, this article will function as a substantiated how-to for future cognitive interviewers.

This article is structured as follows: firstly, we give a brief explanation of cognitive interviewing techniques, followed by an explanation of methods and analyses used for our objective of understanding the interviewer effect in this type of pretesting. Our findings are provided in the order of the interviewing process steps. At the end, we answer the research questions and offer suggestions for improvement.

\subsection{Cognitive interviewing techniques}

Considering different characteristics of questions or different survey requirements, there are several cognitive interviewing techniques used by researchers. Mohorko 
and Hlebec (2013) made a comprehensive overview of cognitive interview techniques as listed by a large number of authors. As they show most methodologists disagree about which techniques should actually be classified under "cognitive interviewing"-some of them include techniques that are not actually cognitive in nature, ${ }^{4}$ others are not comprehensive and/or detailed enough. In this article, we use the classification by Groves et al. (2009); it was adapted from a longer list by Jobe and Mingay (1989), and corresponds to the form of traditional cognitive probes as described in Beatty et al. (1997):

Think-aloud. Respondents verbalize the thoughts they were having while responding to a survey question. They can do it either during (concurrent) or after answering the question or questionnaire (retrospective). Since the interviewers' only role is reading the questions and reminding respondents about their task, their training requirements are minimal, and the technique is mostly free from interviewer-imposed bias. Because of the unusual task of thinking aloud, most of the burden is on respondents, who need preliminary training, and still the success is not guaranteed-respondents often tend to resist or stray from the task.

Verbal probing. Because of the disadvantages of the think-aloud technique, a less strict verbal probing technique has come to extensive use. Respondents answer follow-up questions in order to reveal their response strategies, which shifts the control over the interview to the interviewers and, consequently, lowers respondents' burden. This technique requires more interviewer training and potentially biases the answers, since additional probes may lead to different responses than would be collected in a real survey setting.

Paraphrasing. Respondents demonstrate their understanding of the question by restating it in their own words. Potential problems with this technique include the effects of age and education on the performance of the task.

Definitions. Respondents provide definitions of important or potentially problematic terms in the question.

The authors also include confidence ratings (respondents assess their confidence in their answers); however, this technique is rarely used because of its redundancy. Some authors (e.g., Campanelli et al. 1991; DeMaio and Rothgeb 1996; Oksenberg et al. 1991) showed that respondents' ratings do not correlate with the actual accuracy of their answers and, instead, suggest using definitions or paraphrasing.

Even though the techniques suggested by Groves et al. seem exhaustive enough, we later show that they are not always clear and simple to perform, for either interviewers or respondents.

\section{Methods}

In the following paragraphs, we will explain the methodology used for this study: we will describe the instructions given to interviewers, present an overview of the data collected, and explain the coding of the transcripts for the analysis.

\footnotetext{
4 Those techniques do not focus on mental processes.
} 


\subsection{Instructions for interviewers}

Between the years 2006 and 2010, 120 methodology students ${ }^{5}$ at the University of Ljubljana assessed the quality of assigned survey questionnaires with cognitive interviewing techniques. The students came from all four years of the course and were-for the purpose of the analysis-later split into two groups: the "less experienced" (first and second years) and the "more experienced" (third and fourth years). ${ }^{6}$. Despite the different levels of their previous experiences, they all received the same training in class and detailed instructions on their task during the course. The instructions included:

- General literature on survey quality;

- Specialized reading materials on cognitive interviewing;

- Explanations of techniques in class and support throughout the entire research process;

- An example of data analysis for a particular technique;

- An example of interview transcription.

\subsection{Data analysis}

In the second part of the study, the authors analyzed the interviewers' work. Based on their reports and interview transcriptions, we examined the quality of their work and the degree to which the techniques were understood and correctly realized; we especially focused on the observed problems- either stated or overlooked by the interviewers. We were thus able to understand how successfully a first-time cognitive interviewer would manage to assess the quality of a questionnaire. The program used for the analyses was $\mathrm{NVivo}^{7}{ }^{7}$.

The qualitative analysis consisted of the following stages: First was the initial reading of the materials. Although their quality ranged from very good to very bad, we did not omit any interviews from the analysis, since many findings from those show potential problems for actual cognitive interviewers. Then, assignments were unified in both appearance and structure, without influencing the content. Finally, the reports and transcripts were coded into key analytic categories that were used to illustrate themes and make comparisons and contrasts. Informative examples from this last step will be used in the following sections and referred to in the following order: (student number, questionnaire, technique).

The questionnaire quality assessment using cognitive interviews is usually performed in two main parts: the interview and the analysis of the results. The latter can be conducted either by the interviewers themselves or by other researchers. Since the interviewers in our study were required to perform the entire process, we qualitatively evaluated their work on both stages.

5 These students were studying towards a combined degree in methodology, statistics, and social informatics, and were thus relevant testers, since these experiences and results will be useful to them in their future field of work.

6 All students encountered cognitive interviewing for the first time. Here, we are referring to experience in survey methodology.

7 NVivo10 is a research software for qualitative data analysis 
The quality of the interviewers' work was evaluated using several criteria that will be stated in chronological order of how they (usually) appear in the interviewing process; the results will be structured similarly.

Before the session:

- Effect of previous experience in survey methodology;

- The interviewer's ability to prepare and relax the respondents-their interviewing style.

\section{Starting the interview:}

- Adequate understanding of techniques and ability to improve with practice;

- Whether they performed the test interviews and training, and how well they used the information they had learned;

- The type and amount of motivation they provided to respondents throughout the entire process.

\section{Interviewing process:}

- Adequacy of respondent selection (taking into account their demographical characteristics and behavior during the process);

- Accidental and deliberate mixing of different techniques;

- Particularly noteworthy ideas or mistakes.

After the interview / Analysis:

- Interviewers' personal opinion on the quality of their performance;

- General quality of the meta-analysis;

- Quality of suggestions for improvements;

- Ability to extract information from the interviews;

- Personal opinion on the quality of their meta-analysis;

- Overall evaluation by the authors.

It should be noted that the reports were not standardized; consequently, this information was not equally available by all of the interviewers. Still, because of the integrity of the assignments, we were able to adequately evaluate each student's work.

\subsection{Data overview}

120 interviewers worked with 612 respondents (who were assured of their anonymity) to evaluate 17 different questionnaires ${ }^{8}$ (in Slovenian) that had all been previously used in market or national research. Topics ranged from very impersonal ones (e.g., a specific brand of bathing suit, neighborhood cohesiveness) to very personal ones (e.g., intimate relationships, Internet dating). In Table 1, we provide the number of interviews executed per technique and the abbreviations that will be used in the examples of interview transcriptions.

\footnotetext{
8 For a complete list of used questionnaires, refer to the Appendix.
} 
Table 1 Interviews per technique

\begin{tabular}{lcc}
\hline Technique & Abbreviation & Number of interviews \\
\hline Concurrent think-aloud & cTA & 247 \\
Retrospective think-aloud & rTA & 97 \\
Verbal probing & VP & 96 \\
Definitions & Def & 140 \\
Paraphrasing & Par & 32 \\
\hline Count & & 612 \\
\hline
\end{tabular}

Interviewers had approximately two months to carry out, transcribe, and analyze the interviews (ca. five interviews per interviewer). In the analysis, they described all the questions that were found to be problematic for respondents and provided suggestions for their improvement. Additionally, they had to report their understanding of cognitive interviewing and the used technique, as well as all the difficulties, positive experiences, and interesting respondents' behavior they encountered during their assignment.

\section{Results}

In the following paragraphs, we will thoroughly describe the interviewers' work on the assignments, while focusing on the problems stated in the research questions. We will show that each step in the cognitive interview process is important for the quality of the final results; therefore, the order of our findings will be given according to specific components of the interviewing process: the part before the interviewing session, the start of the interview, the actual interviewing situation, and the analysis part after the interview is finished. However, these parts are never fully independent; therefore, some overlap between different parts of the process will appear in this section. Each part will be concluded with a brief summary of findings-these will be explained more thoroughly in the conclusions.

\subsection{Before the session}

When the interviewers are not also the authors of the questionnaire, they should thoroughly consult with the authors (methodologists) about the aim of the study, the topic, the questions, and response options before beginning the interviews. An example from our research shows that a pair of interviewers working on the same questionnaire noted an important threat to pretesting quality by understanding the concept of "depression" differently, both from their respondents as well as from each other. Whereas one defined depression as a disease $(92, \mathrm{ESS}, \mathrm{VP})$ and commented on all of her respondents understanding the term incorrectly, the other defined depression as a temporary mental state (91, ESS, Def), while all of her respondents understood it as a disease. Still, one of the two stated that, technically, the question is formed accurately, ${ }^{9}$ even though all of her respondents understood it incorrectly. This obviously shows the importance of pretesting even for questions that appear unambiguous. Similarly, interviewer 91 concluded that another

\footnotetext{
9 "Please tell me whether you have felt depressed in the last week."
} 
Table 2 Percentage of successfully executed interviews per level of experience

\begin{tabular}{lcc}
\hline & Less experienced & More experienced \\
\hline Good & 34.1 & 35.0 \\
Average & 43.9 & 37.5 \\
Bad & 22.0 & 27.5 \\
\hline
\end{tabular}

question was formed accurately because all of her respondents understood it the same way. It is clear from the previous example that agreement among respondents does not necessarily imply actual correct understanding. Another example of why discussing the questionnaire with its authors beforehand is necessary can be seen from the following comment:

Question: "How important do you find the characteristic of being a good parent in your partner?" (confusingly stated in Slovene, it could mean both "being good parents" and "having good parents") "I warned the respondent that the question is asking about the partner's parents and not about the partner him- or herself; however, I started having doubts myself later. Mathematically, five people (four respondents and I) interpreted these terms as characteristics of the partner's parents and only one as characteristics of the partner, but this one is enough to start doubting the purpose of the question." (111, Student questionnaire on partnerships, cTA)

\subsubsection{Previous experience}

61 interviewers were classified as less experienced and 59 as more experienced considering their study year and, thus, level of education. We assumed that more experience (i.e., more courses in methodology and statistics) would positively affect the interviewing success; however, as the data in Table 2 show, there is no relation between the two. The quality of the interviewing success was measured on the scale from "good", where the assignment was executed entirely correctly, to "bad", where the assignment was entirely wrong.

The lack-of-experience effect can be assigned to the fact that none of the interviewers had any knowledge in actual cognitive interviewing. This interviewing style is clearly so different from the regular interviews and surveys that previous experience does not apply.

However, despite no trends showing, the influence of other methods on the interviewing style was occasionally obvious: for example, interviewers were interchanging the think-aloud situation with a regular survey setting either excessivelyby behaving like in a regular survey-or not sufficiently-by being too involved when this was not required by the technique. On the other hand, some interviewers with less experience were so insecure in their capabilities that they studied the techniques much more extensively than the experienced ones and, consequently, succeeded:

"Since I had absolutely no experience in interviewing, let alone cognitive interviewing, this seemed a very difficult challenge for me. I prepared for the assignment 
by familiarizing myself with the research method, searching for participants and arranging meetings with them, and, of course, executing the first (test) interview. I read the literature on cognitive interviewing and concurrent think-aloud techniques extensively beforehand; therefore, I had no significant problems (except technical ones)." (34, Authoritarian relationships in the family, cTA)

\subsubsection{Interviewing style}

Table 3 Percentage of successfully executed techniques according to interviewing style

\begin{tabular}{llccc}
\hline & & \multicolumn{3}{c}{ Interviewer } \\
& & Active & Neutral & Passive \\
\hline \multirow{4}{*}{ Technique } & Good & 75.7 & 27.2 & 8.5 \\
& Average & 17.0 & 36.4 & 16.5 \\
& Bad & 7.3 & 36.4 & 75.0 \\
\hline
\end{tabular}

Whereas previous experience did not significantly affect the interviewers' performance, their behavior did. Regardless of the interviewers' sex, interviews were most successful when they were enthusiastic and communicative, understood the technique, and knew how to convey it to respondents. This was clear from the way respondents reacted to their (either passive or active) interviewers and engaged in the conversation, and their opinion on the entire interviewing experience. We qualitatively assessed interviewers' performances according to their transcripts and reports, and coded them as "active" (i.e., they performed above average, were energetic, enthusiastic, and motivated), "passive" (i.e., they performed below average, and appeared bothered and uninterested), or "neutral" (i.e., they performed decently but did not stand out). Cross-referencing their activity with the quality of technique execution (Table 3) shows the importance of an active interviewer for the overall quality of the results: while more than three quarters of active interviewers performed very well, only $8.5 \%$ of the passive ones succeeded. Similarly, only a small number of above average interviewers performed badly. Most of the average-to-bad interviews were performed by a neutral or passive interviewer.

\subsection{Starting the interview}

Approximately half (52) of the interviewers performed an additional test interview before the actual assignment. Nineteen of them explicitly reported benefits of this initial testing-they came to know and understand the technique more profoundly, could better explain it and respond to participants' questions-whereas none mentioned disadvantages of the test interview. Thus, although the test interview does not guarantee the correct execution of the technique (we will explain the most common mistakes in the following paragraphs), it does not compromise the quality of the results.

"Before the actual interview, I carried out a test interview, which helped me acquaint myself with the technique of concurrent think-aloud and other technical 
factors, such as taping with a Dictaphone, writing transcriptions, preparing the respondent to be able to think aloud, etc. I did not have significant problems with the test interview, since my test respondent was communicative and helped me out a great deal." (55, Name generators, cTA)

Within each interview, respondents were prepared for the unusual task of cognitive interviewing with "warm-up questions". Those interviewed with one of the think-aloud techniques answered the classical example question, "Try to visualize the place where you live, and think about how many windows there are in that place. As you count up the windows, tell me what you are seeing and thinking about" (Willis 1999); those involved in the pretesting with the definition or paraphrasing techniques defined a word connected to their questionnaire (e.g., define the word "friendship" or restate the question "How happy do you think you are?"). Thirteen interviewers explicitly stated that these warm-up questions greatly helped both the respondents, who understood the kind of answering expected from them, as well as the interviewers, who learned the kind of responding they should require; whereas nobody mentioned any disadvantages of the warm-up questions.

One problematic factor many interviewers noted was the usage of the recording device. ${ }^{10}$ Although 20 interviewers did notice a change in their speech style (they did not speak as they usually do), respondents did not generally oppose recording. In only 26 cases (4\%), the Dictaphone was reported to have such a strong effect on the respondents that the interviewers genuinely feared for the quality of responses. However, in general, respondents did not refuse participation because of the recording device. Two interviewers reported more than half of their respondents refusing participation, but since they both had such a large number of refusals, it is safe to assume that they did not sufficiently explain the anonymity and privacy of the recordings.

One interviewer had technical problems with his cellphone and had to hold it close to the respondents, which made them very uncomfortable and reserved (50, Relationships, rTA). Five interviewers mentioned fear of answering incorrectly in almost all of their respondents, one of them noting that "... when I turned the Dictaphone on, [the respondents] became silent, changed their tone and speech style, and started to think much more about their answers" (7, Eating habits, rTA)

Apart from the above exceptions, interviewers noticed very similar behavior in their respondents. Thirty respondents were reported to have remained tense and insecure (because of the taping device or the interviewing situation in general) throughout the whole interview. However, most often, they were initially bothered but relaxed after the interviewer hid the taping device, explained that the interviews were anonymous, and that the recordings will be used exclusively for transcriptions, after which they will be destroyed; or simply after the interview began.

\footnotetext{
10 All students used either a Dictaphone, a mobile phone, or a recording computer program to sound-record the sessions.
} 
3.3 The interviewing situation

\subsubsection{Familiarity}

Interviewers were required to interview approximately five respondents; if possible, heterogeneous in demographical characteristics. ${ }^{11}$ Although most chose respondents they personally knew ${ }^{12}$ this was not a guarantee for a successful interview. As we noticed, familiarity was reported as both an advantage (22 cases)respondents trusted the interviewers, felt at ease even with very personal questions, and/or felt obliged to cooperate-as well as a disadvantage (5 cases), either for feeling embarrassed because of the intimate nature of the questions or because they did not take the assignment seriously.

"In my opinion, the reason for the uneasiness was the fact that the respondent and I are not very close; therefore, he did not want to describe his thoughts to me, especially when the question was very unpleasant, personal, or sensitive (e.g., on the issue of mouth odor and furred tongue)." (7, Eating habits, rTA)

"Since I personally dislike discussing private things with strangers, it was very difficult for me to ask for answers to personal questions ..." (54, Name generators, cTA)

"All of the respondents were my acquaintances or neighbors; therefore, I sometimes had the feeling that they were not answering sincerely and were slightly reserved because I was their acquaintance and most of them did not want to expose the problems in their partnerships." (95, Menial tasks, VP)

"He was not really a relaxed respondent; he answered the questions quickly and did not want to cooperate and discuss more in depth with me. He was also the only one of my respondents that always refused answering surveys in the past. He probably only took part in this interview because he is an acquaintance of mine." (94, Menial tasks, VP)

Considering the fact that $22.5 \%$ of the interviewers reported familiarity influencing the interview process, we assume that there is a reasonably strong connection between the two; however, it proved to work as both an advantage and a disadvantage. To assure the former, the interviewer should try to select enthusiastic and communicative respondents, and be able to engage and motivate them.

The process quality was also partly influenced by the type and length of the questionnaire (the more boring ones yielded less excitement than the interesting ones), but not considerably (as shown in a overview of respondent complaints in Table 4).

Thus, the topic of the survey did not have an overall effect on the success of the interviews. Indeed, respondents tended to be more often bored with uninteresting questionnaires, more confused and irritated with difficult ones, and more reserved with intimate ones; for example:

\footnotetext{
11 Sex, age, location, education.

12 Of 66 interviewers that described their choice of respondents, only seven mentioned selecting strangers, and it is safe to assume that a similar trend holds for the rest of the interviewers.
} 
Table 4 Respondents' complaints during the interview

\begin{tabular}{lcc}
\hline Type of complaint & Amount & Percentage of all complaints \\
\hline Length of the questionnaire or interview & 6 & $18.2 \%$ \\
Uninteresting topic/technique & 12 & $36.4 \%$ \\
Characteristics of respondent & 15 & $45.4 \%$ \\
\hline
\end{tabular}

"For the participants as well as for me, the interviews were rather tiresome, since the questions were very much alike or repeated themselves with only slight changes. Consequently, I felt that the respondents were unsatisfied and could not concentrate." (41, The perception of life-path planning, Def)

"With the continuation of the interview, most of the respondents relaxed; however, there was still embarrassment present when questions became more intimate. I think that a female interviewer would be more appropriate for this questionnaire, since the conversation would be easier. ... At one point, I even had to stop the interview, since she was gesturing, indicating that she wanted to stop the conversation." (44, Brand of bathing suits, rTA)

However, while the first example conveys valuable information about the quality of the questions, both cases show the importance of an interviewer's ability to motivate respondents. 120 interviewers were analyzing 17 different questionnaires with no obvious pattern of which questionnaires would gather more positive or more negative attitudes. There was even a case of a respondent that willingly answered a questionnaire on the intimate topic of personal health, despite there being two deaths in his family in that same week (98, ESS, rTA). This implies that the pleasantness of the interviewers and the collaboration between them and the respondents are more important for the success of an interview than the topic.

\subsubsection{Demography effects: respondent}

Demographical traits did have some effect on the interviewing process. Women tried to cooperate much more than male respondents (four interviewers mentioned this occurrence), although sex did not have an effect on which of the two spoke more. Of all the demographical characteristics, age was most often reported to affect the interview, with both the youngest and the oldest respondents. The latter has been repeatedly confirmed in studies (e.g., Dornburg and McDaniel 2006; Holliday 2003; Jobe et al. 1996; Wright and Holliday 2007), along with the (also common) education effect; on the other hand, there are no studies confirming significant sex effect. Two interviewers described age as simply a factor that influenced the respondents' way of thinking and expressing themselves:

"Within the age groups, I noticed differences regarding the definitions of some words; mostly, differences were apparent in thinking about general, abstract concepts that older people perceive differently than students." (30, Questionnaire on social networks, Def)

"Respondents belonged to different age groups, which was obvious from the differences in their thinking-most often on the topic of their values and desires." (9, Neighbourhoods, cTA) 
Nine interviewers emphasized youth being a negative factor with their respondentsbecause of either their childishness or ignorance.

"... His 'immaturity' and irresponsibility due to his youth were obvious from the start. His answers differed from those of other respondents; his teenage way of thinking was evident." (102, ESS, cTA)

"... Lili's responses stood out the most. It should be worth considering rethinking the purpose and the target population, since Lili, being a 15-year-old, has a very different perception of certain terms compared to the other respondents." (42, The perception of life-path planning, Def)

Finally, interviewers mentioned old age having an effect on the responses, both positive (5 occasions) and negative (5 occasions). Older respondents were occasionally confused by the interviewing style they were not accustomed to, while, at other times, endeavored largely to collaborate, which sometimes negatively affected the outcome (since their responses would probably be very different, were they taking a regular survey). Studies show that older respondents might require a different style of interviewing because of their lessened ability of recall (e.g., Dornburg and McDaniel 2006; Jobe et al. 1996; Wright and Holliday 2007); however, obviously, this does not apply to all the elderly, as can also be seen from the following examples:

"Through interviewing, I discovered that some questions contain terms that the older generation does not understand, whereas they are obvious to the younger generation; for example, the word 'bikini'." (47, Brand of bathing suits, Def)

"In my experiment, it was easiest to collaborate with older respondents; they were able to elaborate their thoughts and describe their answers using the retrospective think-aloud technique easily. I have to admit that I was pleasantly surprised because I had expected that they would be the most problematic ones." (51, Relationships, rTA)

Education effect was reported seven times; the interviewers noticed that university students and graduates understood the assignment quicker and were able to collaborate more constructively than those less educated; with one exception:

"Janez, who is the least educated, was the most focused on the survey and gave weighty responses to the questions. On the other hand, Karmen, who has the highest education, was negligent in some questions and lost concentration during the interview." (98, ESS, rTA)

This example suggests that, although education helps understand the techniques and the whole cognitive interviewing process, respondents' willingness to cooperate and be helpful might sometimes be equally (if not more) important.

Finally, there was no particular location (urban vs. rural) effect, apart from the fact that rural residents were most often the interviewer's family members (potential age effect), whereas urban residents were most often friends or other students (potential education effect). 
Table 5 Respondent demography effects per amount of occasions reported

\begin{tabular}{lrr}
\hline Characteristic/Effect & Positive effect & Negative effect \\
\hline Sex & Females (4) & \\
Age & Youth (9) & Old age (5) \\
& Old age (5) & Higher (1) \\
Education & Higher (5) & Lower (1) \\
Location & \multicolumn{2}{c}{ No effect } \\
\hline
\end{tabular}

However, despite Table 5, respondent selection should be based upon the requirements of the study and the specific topic of the questionnaire.

\subsubsection{Demography effects: interviewer}

The effects of the interviewer were measured on a smaller set of demographical variables: sex and level of experience according to their year of study.

The interviewers' sex had some effect on the interview's success. Out of 120 interviewers, ${ }^{13}$ more than a third successfully understood and carried out the technique correctly. However, as shown in Table 6 , female interviewers were slightly more thorough than the men. While there were more women that understood the assignment correctly from the beginning, there were more men overall that performed at least well, implying that knowledge of the techniques can be improved with practice.

Table 6 Percentage of successfully executed techniques per interviewer's sex

\begin{tabular}{lll}
\hline Technique & Female & Male \\
\hline Good & 40.4 & 36.7 \\
Average & 25.0 & 33.3 \\
Bad & 34.6 & 30.0 \\
\hline
\end{tabular}

The effect of the interviewer was also clear in the case of the respondents. A common complaint about cognitive interviews and think-alouds, specifically, is that respondents tend to talk too much and/or often veer off subject. In our case, there were no such occurrences-even very communicative respondents understood that they should focus only on the topic in question. There were many notions of more or less pleasant respondents; yet, our analysis shows that, apart from rare exceptions where respondents stood out because of their unwillingness to cooperate or their exceptionally good understanding of the assignment, most of the responsibility for answer quality fell on the interviewers. Their ability to instruct and stimulate respondents during the process was obvious in the interview transcripts, as well as in the fact that similar successfulness was shown in all

$1337 \%$ male and $63 \%$ female. 
respondents belonging to a particular interviewer. On the other hand, examples where the interviewer could not successfully subdue the respondents ("'Am I easily upset?'-what kind of questions are these? Jeez" [28, Questionnaire on social networks, cTA]; "Am I not allowed to keep anything to myself? This topic is too deep for me"; "No comment"; and, "When is this stupid interview going to end?" [different respondents of interviewer 73, ESS, Def]), despite their responses sometimes being of relatively high quality, show why a selection of adequate participants is equally as important as any other part of the pretesting process.

\subsubsection{Techniques}

At the beginning of this article, we mentioned that the current classifications of cognitive interviewing are not ideal. Of the ones that include techniques referring only to cognitive processes, the classification by Groves et al. (2009) was, in our opinion, the most comprehensive. However, our experiment shows that the interviewers had many difficulties understanding and differentiating between them. In more than half of the cases of concurrent think-aloud, the interviewers and respondents shifted to a regular-type interview at one question, at the least-sometimes to the point of not even giving an actual answer to the survey question, as we see in the following example:

When you are prescribed a medicine, how often do you worry about side effects? Please use this card.

1 - Never or almost never

2 - Some of the time

3 - About half of the time

4 - Most of the time

5 - Always or almost always

6 - (I don't use prescribed medicine)

8 - (I don't know)

Respondent 1: "I trust doctors and their ability to prescribe the appropriate medicine; I am not afraid, but I always read the directions for use first and save them just in case."

Respondent 2: "I don't. (thinking) Except, I remember that I once received very powerful medications, and when the doctor listed all of the side effects, I became slightly scared. But then, nothing awful happened, only a gentle headache."

Respondent 3: "I've never had any problems with bad side effects. I don't use much medicine anyway, very rarely."

Respondent 4: "Always. True, the medicine was tested on hundreds of people, but it has a different effect on each one. And there are always side effects."

Respondent 5: "I'm not afraid, the only thing that is important is that the medicine works correctly." (102, ESS, cTA)

It is obvious that while these replies can qualify as thinking aloud, they do not tell anything about the quality of the question or the adequacy of the response scale, which the interviewer would have to deduce later. In Table 7, we show how many interviewers succeeded in performing an interview with a correctly applied technique using the criteria introduced above. 
Table 7 Amount and percentage of successfully executed techniques by interviewers

\begin{tabular}{|c|c|c|c|c|c|c|c|c|c|c|}
\hline \multirow{2}{*}{$\begin{array}{l}\text { Technique } \\
\text { Good }\end{array}$} & \multicolumn{2}{|c|}{$\begin{array}{l}\text { Concurrent } \\
\text { think-aloud }\end{array}$} & \multicolumn{2}{|c|}{$\begin{array}{l}\text { Retrospective } \\
\text { think-aloud }\end{array}$} & \multicolumn{2}{|c|}{$\begin{array}{l}\text { Verbal } \\
\text { probing }\end{array}$} & \multicolumn{2}{|c|}{ Definitions } & \multicolumn{2}{|c|}{ Paraphrasing } \\
\hline & 17 & 38.6 & 7 & 41.2 & 7 & 29.2 & 15 & 51.7 & 0 & 0.0 \\
\hline Average & 14 & 31.8 & 7 & 41.2 & 11 & 45.8 & 4 & 13.8 & 2 & 33.3 \\
\hline Bad & 13 & 29.5 & 3 & 17.6 & 6 & 25.0 & 10 & 34.5 & 4 & 66.7 \\
\hline
\end{tabular}

Since $82.4 \%$ of the interviews were executed at least averagely, we can conclude that retrospective think-aloud is the most consistent and dependable of all the techniques. If understood correctly, definitions yield the best results, but they can be relatively easily misunderstood (more than a third of the cases). Concurrent think-aloud was only moderately successful, mostly because of the abovementioned fact that the interviewers often shifted to the "regular interview" mode. Verbal probing was a confusing technique both during the interviews, as well as during the analysis of the interviewers' work. The biggest problem with verbal probing is in its loose definition-as Mohorko and Hlebec (2013) show in their overview of cognitive interviewing techniques across different studies, almost all researchers consider some type of probing to be a cognitive interviewing technique. However, while some include very specific probes (e.g., probes about retrieval methods (Akkerboom and Dehue 1997) or scripted concurrent probes (Beatty et al. 1996)), others suggest the umbrella term probe that might represent any type of additional question-including questions that other authors would consider to belong to another technique. This represented a problem for many of our interviewers, as they often switched from this technique to one of the other four (e.g., they asked for a definition or a paraphrase). Since this was technically not wrong, they were coded as "average" in their success; those coded "bad" were entirely wrong in their application of this technique. Lastly, as we will show in the following paragraphs, paraphrasing was clearly the most problematic technique, difficult to understand for both interviewers and respondents.

Apart from the technique swapping due to the unclear definition of verbal probing, unintentional swapping of techniques was a repeated accident (47 occasions); occasionally, both the respondent and the interviewer forgot or confused the technique they were using and, instead, started using another one. Apart from objective reminders to think aloud, interviewers most often asked elaborative probes when they noticed that simply a regular think-aloud interview would not work; on other occasions, interviewers replaced concurrent think-aloud with the definition technique, verbal probing, or retrospective think-aloud. One student described the latter as a consequence of the respondent "becoming too relaxed and unawarely shifting over to the other technique" (56, Name generators, rTA).

Sometimes, the technique switch was deliberate. Two interviewers using the paraphrasing technique (out of six altogether) mentioned having problems with it and were much more pleased with definitions:

"It was almost impossible to paraphrase certain questions; therefore, many candidates repeated them in an almost identical way. Or they simply changed the word order." (22, Neighbourhoods, Par/Def); 
"...[Paraphrasing] turned out rather difficult, since respondents were often unable to transform the question and repeat it in their own words; thus, they usually simply repeated the questions after me and shortened them. ... Paraphrasing was not of much help, so I used the definitions technique, because it was the only way to get to the desired information on how respondents understood the meaning of keywords in the questions; that led me to the understanding of how respondents understand the meaning of the entire question."

"The technique of definitions proved to be more suitable for the respondents and gave more information; unlike with paraphrasing, respondents understood their assignment immediately and had no problems with explaining the term." (all by 21, Neighbourhoods, Par/Def)

These events of technique switching do not necessarily (only) imply the incompetence of the interviewers or respondents, but (also) the inconvenience of some techniques for specific (types of) questions. This would suggest that one questionnaire should not be analyzed with only one technique (however, it could be very confusing for respondents to simultaneously take part in five different techniques). An example showing this necessity is one group's comments on the question Should it be entirely governments' responsibility to ensure a reasonable standard of living for the old?. The set of respondents using definitions defined the word "old" as "older than 50", whereas the group with verbal probing understood the question as referring to pensioners (74, ESS, VP).

In another example, respondents were asked to paraphrase the question on food supplement consumption, whereas the definitions technique would be much more appropriate because of the terminology in the question.

These occurrences suggest that a methodologist should study the questions very carefully before pretesting them, predict the most possible type of problem the question might give rise to, and select the corresponding technique ${ }^{14}$ accordingly.

In general, the interviewers and respondents were most favorable to definitions and verbal probing. They described them as the most straightforward and easiest to understand and explain to respondents, as well as quite interesting.

"All of the respondents agreed that this interview was a great deal more fun than a regular survey and that they have never thought about the exact meanings of some words that seem very obvious but are difficult to explain in one sentence." (109, ESS, Def)

Among all five, these two techniques are the most directly oriented towards seeking possible problems in a questionnaire, which was obvious from the interviewers' suggestions that were much more based on what the respondents actually said. On the other hand, the interviewers with think-aloud techniques or paraphrasing most often suggested corrections in line with their observations of their respondents' struggles. Respondents with concurrent think-aloud also had more problems differentiating between reporting their thoughts and (regular) conversational replying. However, when the execution of the assignment was carried out

\footnotetext{
14 For example, paraphrasing for a wholly vague question, definitions for possibly problematic terms, think-aloud for general or behavior questions etc.
} 
correctly, the student received answers that illustrated the problem with a question much more thoroughly than any other technique would. An example of an accurate concurrent think-aloud:

I consume nutritional supplements/additives: a) daily, b) at least once per week, c) sometimes/rarely, d) I do not consume them.

"Oh, okay, um ... I'm not really sure what exactly nutritional supplements are ... if I count vitamins and similar ... let's say ... once per month. That would be ... c, rarely." (14, Eating habits, cTA)

It was noticeable that many of the interviewers grasped the idea behind cognitive interviewing and fully comprehended the advantages of pretesting only during their last interviews. Table 8 shows the point in the interviewing process where the interviewers were able to grasp the idea of the technique (according to our estimation), where "beginning" represents the time before the first (non-test) interview, "middle/end" represents the time between the first and the last interviews (and implies improvement), and "not at all" represents full incomprehension. ${ }^{15}$ Considering that the improvements were already obvious after their first few tries, it is a clear notion of how practice makes perfect even without initial extensive training, although the interviews were still far from perfection. For higher quality outcomes, we therefore recommend assisting in and performing a few practice interviews before doing the real testing.

Table 8 Amount and percentage of interviewers understanding the technique according to the time point in the research process

\begin{tabular}{|c|c|c|c|c|c|c|c|c|c|c|}
\hline \multirow{2}{*}{$\begin{array}{l}\text { Time point } \\
\text { in interview } \\
\text { Beginning }\end{array}$} & \multicolumn{2}{|c|}{$\begin{array}{l}\text { Concurrent } \\
\text { think-aloud }\end{array}$} & \multicolumn{2}{|c|}{$\begin{array}{l}\text { Retrospective } \\
\text { think-aloud }\end{array}$} & \multicolumn{2}{|c|}{$\begin{array}{l}\text { Verbal } \\
\text { probing }\end{array}$} & \multicolumn{2}{|c|}{ Definitions } & \multicolumn{2}{|c|}{ Paraphrasing } \\
\hline & 20 & 45.5 & 9 & 52.9 & 9 & 37.5 & 15 & 51.7 & 2 & 33.3 \\
\hline Middle/End & 12 & 27.3 & 4 & 23.5 & 7 & 29.2 & 6 & 20.7 & 2 & 33.3 \\
\hline Not at all & 12 & 27.3 & 4 & 23.5 & 8 & 33.3 & 8 & 27.6 & 2 & 33.3 \\
\hline
\end{tabular}

\subsection{After the interview}

Despite the problems that the interviewers had during their interviews, they mostly provided good and useful suggestions for the questions' improvement. We coded the quality of their suggestions and their overall analysis of their interviews again on the scale from "good" to "bad". Table 9 shows that, while almost half of the less experienced interviewers analyzed the results very well, the larger part of the rest performed relatively badly. The more experienced group was slightly more consistent in the sense that they were evenly spread across all three categories.

The characteristics we tested did not suggest the type of interviewer that would be the most successful in accomplishing a good interview analysis. Nevertheless, we

\footnotetext{
15 Tables 7 and 8 are not equivalent, because the latter takes into consideration the ability to learn. In addition, understanding the technique does not necessarily imply good execution, and vice versa.
} 
Table 9 Percentage of well-executed analyses according to previous experience

\begin{tabular}{llccc}
\hline & & \multicolumn{2}{c}{ Previous experience } \\
& & Overall & Less experienced & More experienced \\
\hline \multirow{4}{*}{ Analysis } & Good & 39.0 & 45.0 & 33.3 \\
& Average & 26.8 & 17.5 & 35.7 \\
& Bad & 34.1 & 37.5 & 31.0 \\
\hline
\end{tabular}

suggest that the main analysis be done by the survey author, who is the closest to the research and understands the questions the best. However, even in this case, it is strongly recommended to take into consideration the interviewers' suggestions, regardless of the availability of full transcripts or recordings-both because the interviewer was part of the interviewing situation, as well as because it is simply better to have more constructive opinions than fewer.

\section{Conclusions}

In the previous sections, we presented the outcomes of our longitudinal study, where different techniques of cognitive interviewing were carried out on different types of questionnaires with the purpose of analyzing the interviewer's effect on the success of the interview. The study confirmed that, in the entire cognitive interviewing process, the interviewer is very important, if not the most important factor for a successful interview. We now try to answer our introductory research questions and follow with suggestions for the improvement of interviewer performance and, consequently, the quality of the cognitive interview.

1. How does the interviewing style of an interviewer influence the quality of a cognitive interview?

As stated multiple times throughout this article, the interviewer should be communicative, friendly, enthusiastic, and able to motivate the respondents, and should not make them feel uncomfortable in any way. He or she should be able to stimulate the respondents, especially when dealing with an uninteresting questionnaire or technique. We observed some effect of the interviewer's sex on the quality of their performances; however, overall, the amount of correctly executed techniques was approximately the same for both groups. Similarly, we found no significant effect of previous experience in survey methodology. Cognitive interviewing is considered more similar to a normal conversation than a (very standardized) survey interview (Schaeffer and Maynard 2006), suggesting that the interviewer's personal characteristics become more important-and also more difficult to train.

2. Are some techniques easier to learn/teach than others?

Cognitive interviews consist of several techniques that differ with different authors. We tested the technique selection suggested by Groves et al. (2009), which was, in our opinion, the most concise and exhaustive. The study showed that most techniques are understandable and relatively easy to learn. The exceptions are paraphrasing and verbal probing. The former was mainly difficult for respondents, who were unable to restate questions in a completely different way. We therefore suggest the use of definitions instead of paraphrasing, 
since-in most cases-the former focus more directly on the problematic parts of the question and yield better results. Verbal probing was problematic for interviewers, since they understood it in very different ways. For more success with this technique, we suggest either making the rules much more specific or allowing for a very wide definition of the technique.

3. How large is the respondent effect on the quality of the results?

One of the specialties of cognitive interviews compared to regular surveys is in respondent selection. This method does not require representative sampling and, therefore, allows for a more adequate choice of respondents for the situation. While they should still possess characteristics that will be important in the target population, the researcher should welcome those that show interest in participating and are open-minded enough to be able to learn the new method rather quickly. Most often, these participants will be more highly educated and younger, but as we have showed, there are always exceptions to this rule. Additionally, many surveys that are being tested by cognitive interviewing are specialized for children, the elderly, foreigners, etc., so demographical differences should not be the most important characteristics to consider.

We now give an overview of our conclusions and additional suggestions for the highest quality of interviewer performance. Firstly, interviewers should be very well instructed on the purpose and execution of cognitive interviews. They should understand the whole interviewing process and analysis; for this, we strongly recommend each aspiring cognitive interviewer attend at least one session with an experienced interviewer before carrying one out themselves. Additionally, the interviewer should collaborate with the research team from an early stage of questionnaire development and be extensively tutored about their expectations. On multiple occasions, our interviewers understood the question differently than many or even all of their respondents, which would not have happened if they had known what the researchers had in mind. Previous surveying experiences are not necessarily an advantage. Cognitive interviewing is rather different from regular interviewing; to avoid influence and interference of their previous knowledge, aspiring cognitive interviewers should thoroughly study the techniques both in theory and by observation.

Cognitive interviewing is still a seldom known method and the probability of a volunteer knowing it is low. Most often, it will be mistaken for a survey interview, which, in reality, requires a very different type of interaction; we strongly recommend using warm-up questions at the beginning of the interview, since they give a good idea of the task to both the respondent and the interviewer, and do not have negative effects. Additionally, respondents are not accustomed to surveys being video/sound recorded. Thus, the method used has to be explained at the time of respondent selection, since taping devices are a necessity in the process of cognitive interviewing, regardless of respondents' discomfort towards them. The following implementations may ease the interviewing process: the respondents should be explained the technique at the beginning, which will allow them to decide whether they even want to collaborate (the participants should be volunteers and, preferably, communicative and enthusiastic), prepare better, and not be under the survey type of pressure. Respondents should also clearly understand they are part of the questionnaire design and not of data collection. It should be made clear to them that the researcher is more interested in their opinion on the ques- 
tions rather than in their answers. The usage of the recording device should be explained in the context of their role as a "survey design aid". Since cognitive interviews do not strive for standardized answers but for subconscious hints triggered by the questions being analyzed, it is necessary to re-listen/re-watch the interviews multiple times. Their anonymity and the fact that the tapes will not become public should be unambiguously stated, as well. While respondents should always be informed about the session recording, we suggest making the recording device as unnoticeable as possible; also, other possible (less intruding, e.g., writing responses down) recording options should not be explicitly offered, except when specifically requested. Finally, what should not be forgotten are basic technical requirements (adequate functioning of the device, batteries, sound, etc.) as well as the interviewer's ability to motivate respondents.

In conclusion, even with the techniques that supposedly require the least interviewer training and involvement (both think-alouds), we noticed the sheer importance of this role; only rarely can respondents be blamed for an unsuccessful interview. For high-quality cognitive interviewing results, the interviewers bear the most pressure-to select suitable respondents; give accurate and detailed information on the technique and the whole interviewing process; stimulate, motivate, and remind respondents to think aloud; make them feel comfortable and avoid giving socially desirable responses; be flexible and think on their feet; and, lastly, correctly interpret the results and identify the questionnaire's weaknesses.

In the introduction, we mentioned the research by Beatty and Willis (2007), which theoretically explained cognitive interviewing, and the work by Willis (2005, 1999), which gives practical and hands-on suggestions on how to perform this method for beginners. In our research, we took a step further and monitored the success of first-time cognitive interviewers on both a quantitative and qualitative range. A large amount of interviewers with adequate background knowledge of methodology described their experience in detail, which allows us to see the most common kinds of mistakes and how to avoid them.

Certainly, our research has its drawbacks. In survey methodology, cognitive interviewers are expected to get more specific training for their project, whereas ours was not as thorough. The comparison between interviewers would be more possible with fewer different (or even only one type of) surveys, although we showed that there are no ideal questionnaires and that the interviewer's personality has a greater effect on respondents' collaboration than the actual topic. The techniques we tested belonged to one of many possible classifications of cognitive interviewing, which means that even more of them could be tested. Respondent selection was biased, since interviewers were choosing people they were acquainted with; a true pretesting situation should consist of adequate participants according to the topic of the survey, which was not always met in our research. Nevertheless, our outcomes hold-survey methodologists and researchers should not underestimate the role of the interviewer, which is much larger in cognitive interviewing than in the usual, standardized surveying.

\section{Appendix}

\section{List of used questionnaires}

- Authoritarian relationships in the family 
- Brand of bathing suits

- Domestic roles

- ESS - European Social Survey 2004

- ESS - European Social Survey 2008

- Eating habits

- Factors of cohesiveness and collaboration development in neighbourhoods

- Gathering on the Internet

- Influence of Facebook on social life

- Internet dating

- Menial tasks

- Name generators

- Questionnaire on social networks

- Relationships

- Student questionnaire on partnerships

- Safe food

- The perception of life-path planning

\section{References}

Akkerboom, H., Dehue, F.: The Dutch Model of Data Collection Development for Official Surveys. Int. J. Od Public Opin. Res. 9, 126-145 (1997).

Akkerboom, H., Luiten, A.: Selecting Pretesting Tools According to a Model of Questionnaire Development with Illustrations Concerning Patient Satisfaction with Medical Care. American Statistical Association (1996).

Beatty, P., Schechter, S., Whitaker, K.: Evaluating Subjective Health Questions: Cognitive And Methodological Investigations. American Statistical Association (1996).

Beatty, P., Schechter, S., Whitaker, K.: Variation in Cognitive Interviewer Behavior - Extent and Consequences. American Statistical Association (1997).

Beatty, P., Willis, G.: Research synthesis: The practice of cognitive interviewing. Public Opin. Q. 71, 287-311 (2007).

Bolton, R.N., Bronkhorst, T.M.: Questionnaire Pretesting: Computer-Assisted Coding of Concurrent Protocols. In: Schwarz, N. in Sudman, S. (ur) Answering Questions: Methodology for Determining Cognitive and Communicative Processes in Survey Research. 37-64. Jossey-Bass Publishers, San Francisco, California (1996).

Campanelli, P.: Testing Survey Questions. In: de Leeuw, E.D., Hox, J.J., in Dillman, D.A. (ur) International Handbook of Survey Methodology. 176-200. Taylor \& Francis Group, New York (2008).

Campanelli, P., Martin, E., Rothgeb, J.M.: The Use of Respondent and Interviewer Debriefing Studies as a Way to Study Response Error in Survey Data. J. R. Stat. Soc. Ser. Stat. 40, 253-164 (1991).

Conrad, F.G., Blair, J.: Sources of Error in Cognitive Interviews. Public Opin. Q. $73,32-55$ (2009).

DeMaio, T.J., Landreth, A.: Do Different Cognitive Interview Techniques Produce Different Results? Methods for Testing and Evaluating Survey Questionnaires. 89-108. John Wiley \& Sons, New Jersey (2004). 
DeMaio, T.J., Rothgeb, J.M.: Cognitive Interviewing Techniques In the Lab and in the Field. Answering Questions. Methodology for Determining Cognitive and Communicative Processes in Survey Research. 177-195. Jossey-Bass, San Francisco, California (1996).

Dornburg, C.C., McDaniel, M.A.: The Cognitive Interview Enhances Long-Term Free Recall of Older Adults. Psychol. Aging. 21 (1), 196-200 (2006).

Ericsson, K.A., Simon, H.A.: Verbal Reports as Data. Psychol. Rev. 87, 215-251 (1980).

Goerman, P.L.: Adapting Cognitive Interview Techniques for Use in Pretesting Spanish Language Survey Instruments. American Statistical Association (2006).

Groves, R.M., Fowler Jr., F.J., Couper, M.P., Lepkowski, J.M., Singer, E., Tourangeau, R.: Survey Methodology; 2nd Edition. Hoboken, NJ: John Wiley \& Sons (2009).

Holliday, R.E.: The Effect of a Prior Cognitive Interview on Children's Acceptance of Misinformation. Appl. Cogn. Psychol. 17, 443-457 (2003).

Jabine, T.B., Straf, M.L., Tanur, J.M., Tourangeau, R.: Cognitive aspects of survey methodology: building a bridge between disciplines. Washington, DC: U.S. Department of Education, National Center for Educational Statistics (1984).

Jobe, J.B., Keller, D.M., Smith, A.F.: Cognitive Techniques in Intervewing Older People. In: Schwarz, N. in Sudman, S. (eds.) Answering Questions: Methodology for Determining Cognitive and Communicative Processes in Survey Research. 197-221. Jossey-Bass Publishers, San Francisco, California (1996).

Jobe, J.B., Mingay, D.J.: Cognitive Research Improves Questionnaires. Am. J. Public Health. 79, 1053-1055 (1989).

Jobe, J.B., Mingay, D.J.: Cognition and Survey Measurement: History and Overview. Spec. Issue Appl. Cogn. Psychol. 5, 175-192 (1991).

Knafl, K., Deatrick, J., Gallo, A., Holcombe, G., Bakitas, M., Dixon, J., Grey, M.: The Analysis and Interpretation of Cognitive Interviews for Instrument Development. Res. Nurs. Health. 30, 224-234 (2007).

Mohorko, A., Hlebec, V.: Razvoj kognitivnih intervjujev kot metode predtestiranja anketnih vprašalnikov. Teor. Praksa. (2013).

Neuman, W.L.: Social research methods: qualitative and quantitative approaches. Pearson, Boston (2008).

Oksenberg, L., Cannell, C., Kalton, G.: New Strategies for Pretesting Survey Questions. J. Off. Stat. 7, 349-365 (1991).

Rho, C., Sangster, R.L.: How Much Can you Trust the Answers You Get Using Cognitive Interviews?, http://www.bls.gov/osmr/pdf/st030270.pdf (2003).

Schaeffer, N.C., Maynard, D.W.: Sounds of Silence: The Structure of Response Latencies in Cognitive Assessments. American Association for Public Opinion Research, Wisconsin (2006).

Schwarz, N.: Cognitive Aspects of Survey Methodology. Appl. Cogn. Psychol. 21, 277-287 (2007).

Snijkers, G.: Cognitive Laboratory Experiences on Pre-testing Computerized Questionnaires and Data Quality, (2002).

Sudman, S., Bradburn, N.M.: Asking Questions. The Definitive Guide to Questionnaire Design. Jossey-Bass Inc., Publishers, San Francisco, California (2004).

Tourangeau, R., Couper, M.P., Tortora, R., Steiger, D.M.: Cognitive Issues in the Design of Web Surveys. American Statistical Association Proceedings (2000). 
Tourangeau, R., Rasinski, K.A.: Cognitive Processes Underlying Context Effects in Attitude Measurement. Psychol. Bull. 103 (3), 299-314 (1988).

Willis, G.B.: Cognitive Interviewing?: A "How To" Guide, (1999).

Willis, G.B.: Cognitive Interviewing Revisited: A Useful Technique, in Theory? Methods for Testing and Evaluating Survey Questionnaires. 23-43. John Wiley \& Sons, New Jersey (2004).

Willis, G.B.: Cognitive Interviewing: A Tool for Improving Questionnaire Design. Sage Publications, Thousand Oaks, California (2005).

Wright, A.M., Holliday, R.E.: Interviewing cognitively impaired older adults: How useful is a Cognitive Interview? Memory. 15 (1), 17-33 (2007). 